\title{
Extended Value-Added Intellectual Coefficient and Financial Reporting Quality: Moderating Role of Global Financial Crisis
}

\author{
Dr. Muhammad Irfan \\ Assistant Professor, Institute of Banking \& Finance, Bahauddin Zakariya University, Multan, Pakistan \\ Memoona Kausar* \\ Bahauddin Zakariya University, Multan, Pakistan \\ Dr. Muhammad Shaukat Malik \\ Professor, Institute of Banking \& Finance, Bahauddin Zakariya University, Multan, Pakistan
}

\begin{abstract}
Besides traditional approaches, this study aimed to construct and validate the new model of intellectual capital and financial reporting quality and created a deeper and better understanding through the use of rigorous secondary data. It adopted new formula of intellectual capital instead of traditional primary measures and explored the role of financial reporting quality in financial success of firms. Study is helpful for decision makers, especially from the manufacturing sector, to mould their practices accordingly and they will also realize the importance of high financial reporting quality. The study used Three-stage least squares regression analysis to investigate the relationship between variables. Extended Value-Added Intellectual Coefficient method is used to measure intellectual capital and discretionary revenues method is used to measure financial reporting quality. Panel data was collected from 50 firms, included in fortune global 500 companies list from 2007 to 2017 . Findings disclosed that intellectual capital and process capital has a significant impact on financial performance. Human capital and relational capital negatively influence however, innovation capital and financial reporting quality positively influence financial performance. Global Financial Crisis positively moderates the effect of intellectual capital on financial performance but it doesn't moderate the effect of financial reporting quality on financial performance.

Keywords: Financial Reporting Quality, Intellectual Capital, Global Financial Crisis, Financial Performance, Three-stage least squares regression
\end{abstract}

DOI: $10.7176 / \mathrm{RJFA} / 10-10-12$

Publication date:May $31^{\text {st }} 2019$

\section{Introduction}

This study is predominant as the issue of intellectual capital was of great attention for scholars from last ten years (Hamdan, 2018). The purpose of financial reporting is to make available financial facts about the organizations that are useful to lenders, potential and existing investors, and other creditors in making decisions about investing resources to the firms. So, aim of the paper is to test the effect of financial reporting quality (FRQ) and intellectual capital (IC) on financial performance and moderating effect of global financial crisis (GFC) between financial reporting quality, intellectual capital and financial performance of fortune 500 companies. Fortune 500 companies list is ranking of top 500 companies which earned the highest revenue. Companies have many resources and advanced technology. The list is published in Fortune magazine every year. IC is knowledge-intensive intangible assets that included in a corporation that consist of intellectual competencies, intellectual property and intellectual resources (Chen et al., 2014). IC has three main elements; these are structural capital, relational capital and human capital (Jardon \& Dasilva, 2017). Biddle et al. (2009) explained FRQ as accuracy's level in managing information relevant to cash flows of corporations. Low profit corporations do not make fair financial reports and prepare it difficult for financiers to understand the real picture (Lin et al., 2014). Some organizations do not present R\&D expenditures separately in their annual reports and it is frequently part of the human resource development or investment (Phusavat et al., 2011). Inkinen (2015) presented publication frequency of IC's components through the graph and mentioned that innovation capital is the most scarcely discussed element and even process capital is not included in the list. Martinez-Ferrero (2014) examined association of FRQ and firm performance measured by dividing market and book which is a market measure so scholar recommended that accounting measures are required to measure the firm performance. So, test of association of FRQ on financial performance is needed. By literature it is clear that GFC 2007-09 badly disturb financial position of organizations and countries' economy. Financiers can alter their priorities and pattern of resources allocation.

\section{Literature review and Hypotheses Development}

Ding (2010) explored that firstly, John Kenneth Galbraith presented the term of intellectual capital (IC) in 1969. In the definition of IC, Stewart (1997) incorporated information, intellectual property, expertise, education, knowledge and experience. In the perspective of Western European, IC is a vibrant source for the generation of 
firms' value (Sardo \& Serrasqueiro, 2017). Influence of IC on organizational performance is stronger in the knowledge-based firms than the traditional firms in India (Maji \& Goswami, 2016). But Morariu (2014) insisted that IC has an inverse relationship with market value and has an insignificant link with return on equity of Romanian firms. Ozkan et al. (2017) insisted that there is a strong association between financial performance (Return on Assets) and IC of Turkish Banks. IC has a positive effect on organizational performance (Pucci et al., 2015; Khalique et al., 2015). Nevertheless, Study derived that the IC has no influence on firm performance (Earnings per Share, Tobin's Q) of listed Tehran Stock Exchange firms (Vazifehdoust et al., 2014). Moreover, IC has a positive influence on corporate return and profitability (Jordão \& Almeida, 2017). In addition to, IC has a positive relationship with accounting measures (return on assets). However; IC has not correlated to the market performance of corporations in Bahrain and Saudi Arabia (Hamdan, 2018). IC has a strong effect on financial performance (return on assets, return on equity) of banks (Al-Musali \& Ismail, 2016). But, Celenza and Rossi (2014) investigated that there is no positive association between IC, return on investment, market to value, return on equity and return on sales within Italian firms. IC has a strong positive influence on financial performance and market value of non-financial firms of Western Europe (Sardo \& Serrasqueiro, 2017). Similarly, IC has a significant influence on firm's performance of technology industry (Nimtrakoon, 2015), pharmaceutical in India (Sriranga \& Vijay, 2014) and SMEs of Portugal (Ferreira \& Franco, 2017).

$\mathbf{H}_{1}$ : Intellectual capital has a significant effect on financial performance.

Human capital (HC) is a main element for value generation in this knowledge-based era. Human assets consist of expertise, managerial skills, creativity, entrepreneurial, leadership and competence of problem-solving having by the personnal of the organizations (Brooking, 1996). Creative ideas, professional skills, work experience and knowledge increased operational and financial performance of the corporations (Wang et al., 2014). HC positively influence the performance of Russian firms (Andreeva \& Garanina, 2016). Similarly, HC has positive influence on the financial performance of banks of Turkey (Ozkan et al., 2017). But, Chu et al. (2011) described that there is no link of HC with assets turnover of listed firms of Hong Kong. Higher the level of HC, the higher is level of organizational performance of Iranian listed firms (Asiaei \& Jusoh, 2015). But, the association between HC and firm performance is insignificant in Malaysian firms (Hashim et al., 2015). Wang et al. (2014) found a strong positive association between the financial performance and $\mathrm{HC}$ of technology firms in China. Moreover, HC has a strong positive influence on the bank's performance in Luxembourg and Belgium (Mention \& Bontis, 2013). HC has significant positive relationship on firm performance of steel and engineering firms in India (Maji \& Goswami, 2016), Iranian chemical and pharmaceutical industry (Salehi et al., 2014), Malaysian Listed Companies (Abdullah \& Sofian, 2012), Greek firms from different industries (Maditinos et al., 2011).

$\mathbf{H}_{1 \mathrm{a}}$ : Human capital has a significant effect on financial performance.

Structural Capital (SC) is the knowledge that kept with the organiztion when human resource goes home (Roos et al., 1998). SC has a significant effect on performance of firm (Khalique et al., 2015; Gogan et al., 2016; Sriranga \& Vijay, 2014; Cabrita \& Bontis, 2008). SC is divided into relational capital and organizational capital. Organizational capital is divided into innovation capital and process capital ( $\mathrm{Su}$ et al., 2013; Sumedrea, 2013; Bontis, 2001; Cohen \& Kaimenakis, 2007; Namvar et al., 2012).

Relational capital (RC) described as ability of a firm to work together with stakeholders of outside organization e.g. suppliers, competitors, industry associations, customers and trade (Edvinsson \& Malone, 1997). By developing RC, firms may found various better ways of business operating by getting knowledge from other people experiences and can be more innovative (Cousins et al., 2006). RC by using measurements of agencies, agencies' skills and market share has a strong positive impact on organization efficiency of Iranian insurance companies (Zakery \& Afrazeh, 2015). On the contrary, RC has not positive significant influence on the financial performance of the technology industry of five stock exchanges (Nimtrakoon, 2015). RC increases operational and financial performance of technology corporations (Wang et al., 2014). RC has a significant influence on business performance of Romanian drinking water firms (Gogan et al., 2016), non-financial firms of Western Europe (Sardo \& Serrasqueiro, 2017), Iranian chemical and pharmaceutical industry (Salehi et al., 2014), Malaysian Listed Companies (Abdullah \& Sofian, 2012), Portuguese financial services Sector (Cabrita \& Bontis, 2008), Iranian public listed firms (Asiaei \& Jusoh, 2015). However, Andreeva and Garanina (2016) demonstrated that RC has not positive effect on the organizational performance of Russian manufacturing companies, as in Russia RC is so extraordinary that it doesn't present as a competitive advantage.

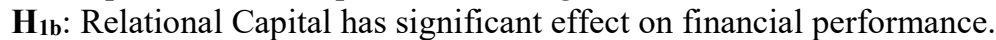

Innovation capital ( $\mathrm{InC}$ ) described as the capability of the corporation to use current knowledge to produce knowledge, new products, ideas and technologies (Maditinos et al., 2010). Nowadays innovation is a requirement for each organization because of the fast growth of technology, globalization and market competition. Innovation can influence the organization's long-term success (Naranjo-Valencia et al., 2016). A corporation can decrease the production cost with technological innovation and gain more profit with product innovation (Chen et al., 2004). Process and product innovation have significant positive whereas marketing and organizational innovation has non-significant effect on corporate performance (Atalay et al., 2013). HC significantly influences InC (Namvar et 
$a l ., 2012$ ). InC has a positive association with performance (return on assets, profitability and return on investments) of Taiwan's IT/electronic industry (Chiou \& Chen, 2012). On the contrary, InC has no impact on market value (Ferraro \& Veltri, 2011). Moreover, Maditinos et al. (2010) mentioned that InC has a positive relation on SC. But, Jiang et al. (2013) discussed that there is an inverse association between innovation performance and partnering with other corporations via alliances. InC has insignificant impact on return on equity, employee productivity and return on assets; on the contrary there is a positive inflence on revenue growth of Thailand firms (Phusavat et al., 2011). Expenses of R\&D has strong linked with the future performance of Chinese corporations (Ruiqi et al., 2017).

H1c: Innovation capital has significant effect on financial performance.

Process capital (PrC) is related to procedures, formulas, work instructions, standards, charts, guidelines and techniques that increase the efficiency of transfer of services and goods to customers (Laitinen, 2009). PrC is the procedures, techniques and systems which a corporation adopts to attain process quality and operational efficiency (Scafarto et al., 2016). PrC can influence firm performance by increasing customer performance and minimizing the cost of operations (Cheng et al., 2010). Moreover, Indicators of national IC, i.e. InC, market capital, PrC and $\mathrm{HC}$ has a strong positive effect on GDP per capita (Phusavat et al., 2012). Moreover, IC with elements of InC, HC, $\mathrm{PrC}$ and customer capital has positive association with innovation, knowledge management, learning organization and self-directed learning (Phusavat et al., 2013). PrC is the main element of IC that affects Taiwan's Public Accountants firms in holding high operating performance (Lee \& Lin, 2018). Similarly, Namvar et al. (2012) indicated that $\mathrm{HC}$ significantly effect PrC in Iranian IT companies. However, PrC has non-significant impact on the market value of Italian listed firms (Ferraro \& Veltri, 2011). Small power distance, individualism and Weak uncertainty avoidance are helpful to the generation of PrC (Lee et al., 2017).

H1d: Process capital has significant effect on financial performance.

Financial reports are information resources available to capital markets and it plays an effective role in investment development (Iatridis, 2010). Peek et al. (2010) mentioned that nonpublic organizations involved in short informative financial transparency than public listed firms. Financial disclosure's quality has non-significant association with stock return of corporation (Salehi et al., 2018). FRQ has a positive significant effect on investment efficiency (Mohammadi, 2014; Chen et al., 2011). Practices of corporate governance have a strong positive effect on transparency of information of non-financial listed firms (Kachouri \& Jarboui, 2017). But, larger firms and ownership concentration have an inverse impact on FRQ (Yasser et al., 2017). In addition to, audit committees play a key role in regulating low FRQ and bring back the user's confidence in financial reports (Salehi \& Shirazi, 2016). FRQ has a strong positive influence on corporate dividend policy (Koo et al., 2017). Nevertheless, FRQ has an inverse association with the family firm's investment behavior and when a member of family acted as the CEO, there is insignificant impact of FRQ on investment decision (Lin et al., 2016). The internal control system's Implementation, internal accountant's competence and commitments of internal managers to the company has a strong effect on the FRQ (Setiyawati, 2013). On the contrary, corporate governance has no significant association with FRQ (Honu \& Gajevszky, 2014). By high FRQ, information asymmetry may be reduced between SMEs and their creditors (Bauwhede et al., 2015). In addition to, there is an inverse influence between FRQ and innovation (Lobo et al., 2018). Kardan et al. (2016) mentioned that FRQ on the basis of qualitative characteristics has a positive influence and on basis of Dechow and Dichev (2002) model, FRQ has an inverse influence on debt financing. Moreover, FRQ has a negative influence on equity financing. After the adoption of IFRS, there is positive association between board governance and accounting information's quality (Krismiaji et al., 2016).

$\mathbf{H}_{2}$ : Financial reporting quality has a significant effect on financial performance.

Global financial crisis (GFC) started in 2007's fourth quarter and continued to disorder the financial system until 2009's second quarter. Dwyer and Lothian (2012) claimed that GFC was in the retrieval stage by 2011. After depression of the 1930s, GFC 2007-08 is called the worst crisis (Reinhart \& Rogoff, 2013). The growth of corporations was affected by $\mathrm{HC}$ and $\mathrm{SC}$ and new concepts and ways for operating firms were emerged and developed during GFC (Sumedrea, 2013). GFC has no link with disclosures of IC in corporations of Portugal (Rodrigues et al., 2017). Market structure and GFC have a significant effect on IC in UAE (El-Bannany, 2012). Because of GFC, countries face long time period unemployment and reduction in consumer's spending, housing foreclosures and business investment (Reinhart \& Rogoff, 2009). Social capital has a positive impact on a firm's stock returns during GFC (Lins et al., 2017). Mostly Thai corporations kept safe from GFC because they don't deleverage and liquidate their assets (Vithessonthi and Tongurai, 2015). Despite independent boards and high institutional ownership, organizations got lower returns of stock during GFC (Erkens et al., 2012). Capital markets progressed in harmonization and stock's prices in world reduce by thirty percent or more during GFC (Bartram and Bondar, 2009). When the crisis increases, banking sector was the most affected sector (Cecchetti, 2009). Leverage has a strong impact on the performance of small enterprises but, there is a negative influence of Leverage on the performance of large corporations during GFC in Thailand (Vithessonthi \& Tongurai, 2015). Kehelwalatenna (2016) explained that there is a worsening of the IC's reputation during the GFC in banks of New 
York.

$\mathbf{H}_{3}$ : Global Financial Crisis has moderating effect between intellectual capital and financial performance.

$\mathbf{H}_{4}$ : Global Financial Crisis has moderating effect between financial reporting quality on financial performance.

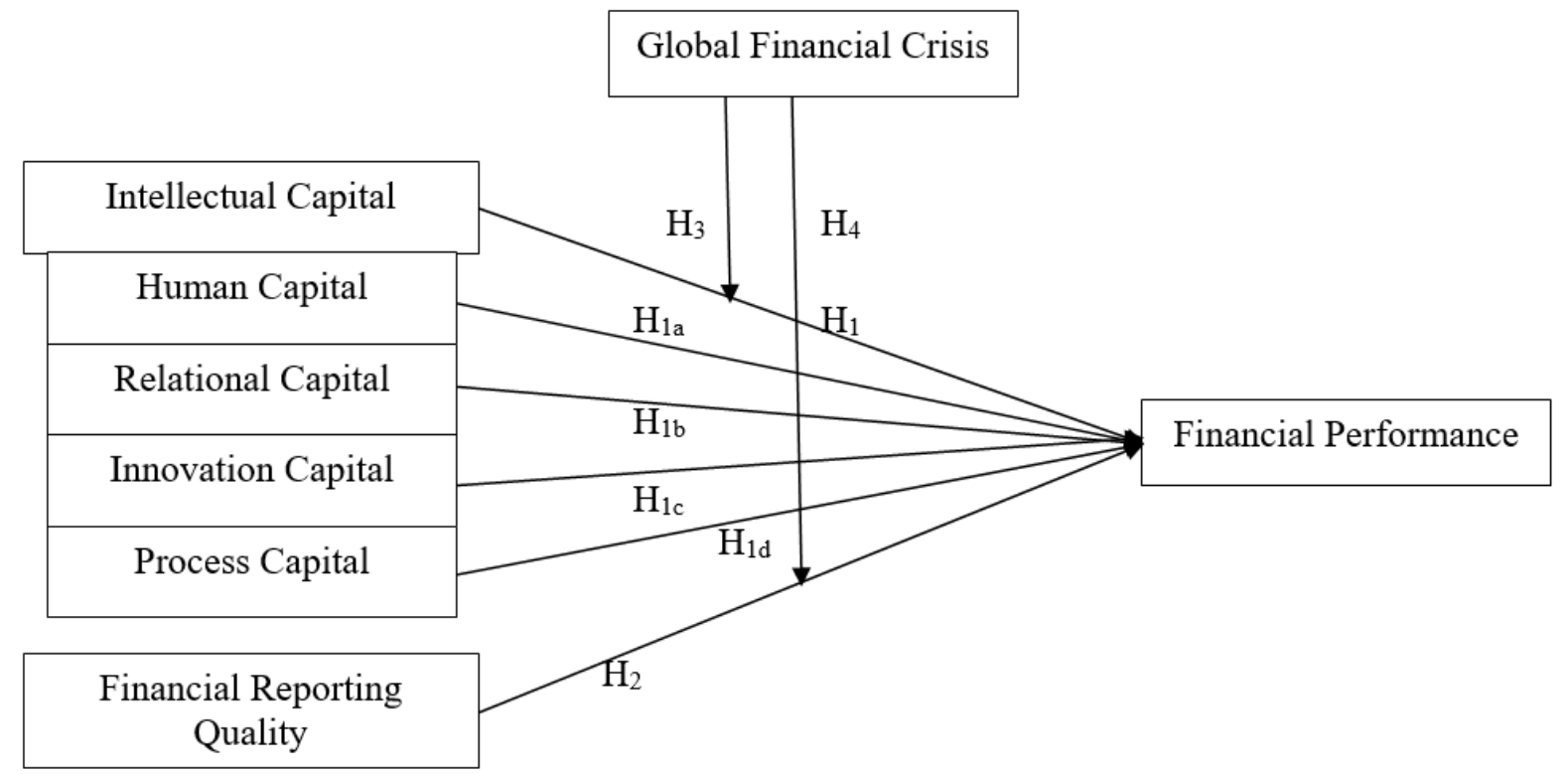

Figure 1: The Research Model

\section{Research Methodology}

This study employed a rigorous research methodology. It is a quantitative research using panel data. For regression analysis of panel data, EViews is used. Research hypotheses are tested using Three-stage least squares regression analysis. For this causal study, 50 companies are selected, of which 17 are from pharmaceutical industry, 16 are from electronics industry and 17 are from chemical industry. Selected companies earned highest revenue during 2016 to 2018. Data is collected of last eleven years i.e. from 2007 to 2017. The financial data is composed from annual financial reports and annual reports are available on firm' official websites. According to law, these financial reports should use standard accounting principles and practices and should be audited. That's why we are assuming that our data is reliable and our results should be verifiable. To measure the intellectual capital efficiency, Pulic (1998) presented a method with parts of financial capital, value added and intellectual capital. This method is named as Value-Added Intellectual Coefficient (VAIC). Many researchers have used VAIC method (Sriranga and Vijay, 2014; Sardo \& Serrasqueiro, 2017; Ozkan et al., 2017; Salehi et al., 2014; Maji \& Goswami, 2016; Singh et al., 2016; Maditinos et al., 2011; Mondal and Ghosh, 2012). So, this method is most reliable method. VAIC $=($ Value Added/Human Capital + Structural Capital/ Value Added $)+$ Value Added /Capital Employed Nazari and Herremans (2007) extended the pulic's VAIC method and integrated innovation, process and relational capital into VAIC model. So, this study used an extended Value-Added Intellectual Coefficient method (eVAIC). eVAIC $=\{$ Value Added $/$ Human Capital $+($ Relational Capital/Value Added + Innovation Capital/Value Added + Process Capital/Value Added) $\}$ + Value Added/Capital Employed

Discretionary revenues method is used to measure financial reporting quality (Yasser et al., 2016; Stubben, 2010). GFC is a dummy variable. GFC is codded 1 during the period of GFC 2008-09 and otherwise 0 (El-Bannany, 2012; Al-Musali and Ismail, 2016).

To measure financial performance, Dividend payout ratio (DPR), Firm growth rate (FGR), Return on capital employed (ROCE) and Employee productivity (EP) is used. The dividend payout ratio is computed by dividing dividend per share to earnings per share (Enekwe et al., 2015). Firm Growth Rate is computed by subtracting logarithm of the total sale of last year from the logarithm of the total sale of the current year (Colombelli, 2015). Return on capital employed is computed by dividing earnings before interest and tax to capital employed (Bhatt \& Bhattacharya, 2015). Employee productivity is computed by dividing profit before tax to a total number of employees (Clarke et al., 2011). Control variables are Firm leverage (FL), firm size (FS) and firm age (FA) in this study. Firm Leverage is computed by the dividing total debt and total assets (Shiu, 2006; Clarke et al., 2011). Firm Age is computed by a number of years since the firm's startup date (Díaz-Fernandez et al., 2015; Hamdan, 2018). Firm size is computed by the natural logarithm of the book value of total assets (Alipour, 2012).

Following regression models are developed in study: 
Table 1: Regression Models

\begin{tabular}{|c|c|}
\hline Model & Regression Equation \\
\hline 1 & $\mathrm{FGR}=\beta_{0}+\beta_{1}\left(\mathrm{FRQ}_{\mathrm{i}, \mathrm{t}}\right)+\beta_{2}\left(\mathrm{eVAIC} \mathrm{i}_{\mathrm{i}, \mathrm{t}}\right)+\beta_{3}\left(\mathrm{FS}_{\mathrm{i}, \mathrm{t}}\right)+\beta_{4}\left(\mathrm{FL}_{\mathrm{i}, \mathrm{t}}\right)+\beta_{5}\left(\mathrm{FA}_{\mathrm{i}, \mathrm{t}}\right)+\varepsilon_{\mathrm{i}, \mathrm{t}}$ \\
\hline 2 & $\mathrm{DPR}=\beta_{0}+\beta_{1}\left(\mathrm{FRQ}_{\mathrm{i}, \mathrm{t}}\right)+\beta_{2}\left(\mathrm{eVAIC} \mathrm{i}_{\mathrm{i}, \mathrm{t}}\right)+\beta_{3}\left(\mathrm{FS}_{\mathrm{i}, \mathrm{t}}\right)+\beta_{4}\left(\mathrm{FL}_{\mathrm{i}, \mathrm{t}}\right)+\beta_{5}\left(\mathrm{FA}_{\mathrm{i}, \mathrm{t}}\right)+\varepsilon_{\mathrm{i}, \mathrm{t}}$ \\
\hline 3 & $\mathrm{EP}=\beta_{0}+\beta_{1}\left(\mathrm{FRQ}_{\mathrm{i}, \mathrm{t}}\right)+\beta_{2}\left(\mathrm{eVAIC}_{\mathrm{i}, \mathrm{t}}\right)+\beta_{3}\left(\mathrm{FS}_{\mathrm{i}, \mathrm{t}}\right)+\beta_{4}\left(\mathrm{FL} \mathrm{i}_{\mathrm{i}, \mathrm{t}}\right)+\beta_{5}\left(\mathrm{FA}_{\mathrm{i}, \mathrm{t}}\right)+\varepsilon_{\mathrm{i}, \mathrm{t}}$ \\
\hline 4 & $\mathrm{ROCE}=\beta_{0}+\beta_{1}\left(\mathrm{FRQ}_{\mathrm{i}, \mathrm{t}}\right)+\beta_{2}(\mathrm{eVAIC} \mathrm{i,t})+\beta_{3}\left(\mathrm{FS}_{\mathrm{i}, \mathrm{t}}\right)+\beta_{4}\left(\mathrm{FL}_{\mathrm{i}, \mathrm{t}}\right)+\beta_{5}\left(\mathrm{FA}_{\mathrm{i}, \mathrm{t}}\right)+\varepsilon_{\mathrm{i}, \mathrm{t}}$ \\
\hline 5 & $\mathrm{FGR}=\beta_{0}+\beta_{1}\left(\mathrm{FRQ}_{\mathrm{i}, \mathrm{t}}\right)+\beta_{2}\left(\mathrm{HC}_{\mathrm{i}, \mathrm{t}}\right)+\beta_{3}\left(\mathrm{RC}_{\mathrm{i}, \mathrm{t}}\right)+\beta_{4}\left(\mathrm{InC}_{\mathrm{i}, \mathrm{t}}\right)+\beta_{5}\left(\mathrm{PrC}_{\mathrm{i}, \mathrm{t}}\right)+\beta_{6}\left(\mathrm{FS}_{\mathrm{i}, \mathrm{t}}\right)+\beta_{7}\left(\mathrm{FL}_{\mathrm{i}, \mathrm{t}}\right)+\beta_{8}\left(\mathrm{FA}_{\mathrm{i}, \mathrm{t}}\right)+\varepsilon_{\mathrm{i}, \mathrm{t}}$ \\
\hline 6 & $\mathrm{DPR}=\beta_{0}+\beta_{1}\left(\mathrm{FRQ} \mathrm{i}_{\mathrm{i}, \mathrm{t}}\right)+\beta_{2}\left(\mathrm{HC}_{\mathrm{i}, \mathrm{t}}\right)+\beta_{3}\left(\mathrm{RC}_{\mathrm{i}, \mathrm{t}}\right)+\beta_{4}\left(\operatorname{InC}_{\mathrm{i}, \mathrm{t}}\right)+\beta_{5}\left(\operatorname{PrC}_{\mathrm{i}, \mathrm{t}}\right)+\beta_{6}\left(\mathrm{FS}_{\mathrm{i}, \mathrm{t}}\right)+\beta_{7}\left(\mathrm{FL}_{\mathrm{i}, \mathrm{t}}\right)+\beta_{8}\left(\mathrm{FA}_{\mathrm{i}, \mathrm{t}}\right)+\varepsilon_{\mathrm{i}, \mathrm{t}}$ \\
\hline 7 & $E P=\beta_{0}+\beta_{1}\left(F R Q_{i, t}\right)+\beta_{2}\left(H_{i, t}\right)+\beta_{3}\left(R_{i, t}\right)+\beta_{4}\left(\operatorname{InC}_{i, t}\right)+\beta_{5}\left(\operatorname{PrC}_{i, t}\right)+\beta_{6}\left(F_{i, t}\right)+\beta_{7}\left(F L_{i, t}\right)+\beta_{8}\left(F A_{i, t}\right)+\varepsilon_{i, t}$ \\
\hline 8 & $\mathrm{ROCE}=\beta_{0}+\beta_{1}\left(\mathrm{FRQ}_{\mathrm{i}, \mathrm{t}}\right)+\beta_{2}\left(\mathrm{HC}_{\mathrm{i}, \mathrm{t}}\right)+\beta_{3}\left(\mathrm{RC}_{\mathrm{i}, \mathrm{t}}\right)+\beta_{4}\left(\operatorname{InC}_{\mathrm{i}, \mathrm{t}}\right)+\beta_{5}\left(\operatorname{PrC}_{\mathrm{i}, \mathrm{t}}\right)+\beta_{6}\left(\mathrm{FS}_{\mathrm{i}, \mathrm{t}}\right)+\beta_{7}\left(\mathrm{FL}_{\mathrm{i}, \mathrm{t}}\right)+\beta_{8}\left(\mathrm{FA}_{\mathrm{i}, \mathrm{t}}\right)+\varepsilon_{\mathrm{i}, \mathrm{t}}$ \\
\hline
\end{tabular}

Note: Model 1, 2, 3 and 4 examine the relationships between IC and financial performance. The last four models (Models 5, 6, 7 and 8) are used to analyze each of the four components of IC on the dependent variable.

\section{Results and Discussion}

This paramount study undertook normality, unit root, heteroscedacity, multicollinearity, outliers, autocorrelation and endogeneity tests to ensure the quality of data and variables.

Table 2: Descriptive Statistics

\begin{tabular}{lcccccccc}
\hline Variables & $\mathrm{N}$ & Mean & Median & Maximum & Minimum & Std.Dev. & Skewness & Kurtosis \\
\hline eVAIC & 547 & 1.5344 & 1.3494 & 3.6721 & 0.3471 & 0.6287 & 0.8381 & 3.3681 \\
HC & 550 & 0.0856 & 0.0801 & 0.3275 & 0.0022 & 0.0657 & 0.6882 & 2.6755 \\
RC & 550 & 0.1284 & 0.1174 & 0.4745 & 0.0074 & 0.0757 & 0.9384 & 4.6196 \\
InC & 550 & -3.3881 & -3.2441 & -1.6550 & -6.3288 & 0.8658 & -0.7988 & 3.3852 \\
PrC & 550 & 1.3484 & 1.3155 & 3.6398 & -0.4243 & 0.6801 & 0.0739 & 3.8552 \\
FRQ & 550 & -4.6822 & -4.5427 & -0.9606 & -11.205 & 1.4215 & -0.7395 & 4.7242 \\
GFC & 550 & 0.1818 & 0 & 1 & 0 & 0.3860 & 1.6499 & 3.7222 \\
FGR & 550 & 0.0199 & 0.0182 & 0.4715 & -0.3095 & 0.0676 & 0.6765 & 10.543 \\
DPR & 497 & 3.6888 & 3.6956 & 9.4173 & -0.5596 & 0.8575 & 0.2942 & 9.8706 \\
EP & 525 & 4.5571 & 4.2899 & 11.215 & -1.6094 & 1.7199 & 1.1126 & 5.5888 \\
ROCE & 539 & -2.1865 & -2.1562 & 0.0048 & -6.2619 & 0.8040 & -0.9933 & 6.7777 \\
FS & 550 & 2.8927 & 2.8662 & 3.1996 & 2.7203 & 0.1105 & 1.2531 & 3.9583 \\
FA & 550 & 86.28 & 95.5 & 215 & 2 & 51.030 & 0.1142 & 2.1847 \\
FL & 550 & 0.6031 & 0.6190 & 1.3186 & 0.2412 & 0.1361 & 0.0756 & 4.6149 \\
\hline
\end{tabular}

Note: As some values of skewness was not lied within recommended range of skewness so adopted log transformation method for extended Value-Added Intellectual Coefficient, innovation capital, process capital, financial reporting quality, dividend payout ratio, employee productivity, return on capital employed and firm size.

Table 2 exhibited the descriptive statistics of the data. The recommended range of skewness is between -1 and +1 (Hair et al., 2006). It is evident from table 2 that values of skewness are between -1 and +1 so after checking normality test, data can be viewed as perfect normal distribution. Charbaji (2011) argues that ratio variables increase skewness in the data so one should log-transform the data for better statistical analysis so, used log transformation where it was needed.

Table 3: Unit Root Test

\begin{tabular}{lcc}
\hline Variables & Statistics & Prob. \\
\hline eVAIC & 198.491 & $0.0000^{*}$ \\
Human Capital & 175.324 & $0.0000^{*}$ \\
Relational Capital & 150.706 & $0.0008^{*}$ \\
Innovation Capital & 152.998 & $0.0003^{*}$ \\
Process Capital & 163.757 & $0.0001^{*}$ \\
Financial Reporting Quality & 171.213 & $0.0000^{*}$ \\
Global Financial Crisis & 203.008 & $0.0000^{*}$ \\
Firm Growth Rate & 217.115 & $0.0000^{*}$ \\
Dividend Payout Ratio & 145.288 & $0.0014^{*}$ \\
Employee Productivity & 177.670 & $0.0000^{*}$ \\
Return on Capital Employed & 210.681 & $0.0000^{*}$ \\
Firm Size & 134.948 & $0.0114^{* *}$ \\
Firm Leverage & 169.057 & $0.0000^{*}$ \\
Firm Age & 381.711 & $0.0000^{*}$
\end{tabular}

Note: $* p<0.01, * * p<0.05$

This study applied ADF - Fisher Chi-square to check problem of unit root in panel data and in table 3, findings 
disclosed that data has no unit root problem (Maddala \& Wu, 1999). Breusch pagan test proved that there is no heteroscedacity problem in the data. Endogeneity test revealed that all variables are exogenous.

Table 4: Correlation Analysis

\begin{tabular}{llllll}
\hline Variables & eVAIC & FRQ & Firm Size & Firm Leverage & Firm Age \\
\hline eVAIC & 1 & & & & \\
FRQ & $0.01613^{* *}$ & 1 & & & \\
Firm Size & $-0.04295^{* *}$ & $-0.02673^{* *}$ & 1 & & \\
Firm Leverage & $-0.02594^{* *}$ & $0.08155^{* * *}$ & $-0.04215^{* *}$ & 1 & \\
Firm Age & $-0.07697^{* * *}$ & $-0.06312^{* * *}$ & -0.19554 & $0.01920^{* *}$ & 1 \\
\hline
\end{tabular}

Note: $* \mathrm{p}<0.01, * \mathrm{p}<0.05, * * * \mathrm{p}<0.1$

Correlation analysis gives assistance to identify the existence of multicollinearity between variables. Multicollinearity should be considered a serious concern only if the correlation among variables exceeds 0.8 (Kennedy, 1985). From Table 4, it is evident that correlation coefficients range from a low of -0.196 to a high of 0.081 .

Table 5: Regression Analysis for Model 1, Model 2, Model 3 and Model 4

\begin{tabular}{|c|c|c|c|c|c|c|c|c|c|c|c|c|c|c|c|c|}
\hline \multirow[b]{2}{*}{ Variables } & \multicolumn{4}{|c|}{ FGR } & \multicolumn{4}{|c|}{ DPR } & \multicolumn{4}{|c|}{$\mathrm{EP}$} & \multicolumn{4}{|c|}{ ROCE } \\
\hline & B & $\begin{array}{l}\text { Std. } \\
\text { Error }\end{array}$ & $\begin{array}{l}\mathrm{t}- \\
\text { Statistic }\end{array}$ & $\mathrm{p}$ & $\beta$ & $\begin{array}{l}\text { Std. } \\
\text { Error }\end{array}$ & $\begin{array}{l}\text { t- } \\
\text { Statistic }\end{array}$ & $\mathrm{p}$ & $\beta$ & $\begin{array}{l}\text { Std. } \\
\text { Error }\end{array}$ & $\begin{array}{l}\text { t- } \\
\text { Statistic }\end{array}$ & $\mathrm{p}$ & $\beta$ & $\begin{array}{l}\text { Std. } \\
\text { Error }\end{array}$ & $\begin{array}{l}\text { t- } \\
\text { Statistic }\end{array}$ & $P$ \\
\hline Constant & 0.528 & 1.687 & 0.313 & 0.755 & 5.174 & 1.152 & 4.492 & $0.000^{*}$ & -25.69 & 1.329 & -19.33 & $0.000^{*}$ & 4.722 & 0.911 & 5.184 & $0.000^{*}$ \\
\hline eVAIC & 0.061 & 0.093 & 0.653 & 0.514 & -0.168 & 0.062 & -2.715 & $0.007^{*}$ & 0.813 & 0.073 & 11.13 & $0.000^{*}$ & 0.248 & 0.051 & 4.816 & $0.000 *$ \\
\hline FRQ & 0.263 & 0.043 & 6.076 & $0.000 *$ & -0.048 & 0.027 & -1.810 & 0.071 & 0.098 & 0.032 & 3.044 & $0.002 *$ & 0.007 & 0.023 & 0.326 & 0.744 \\
\hline FS & -0.956 & 0.549 & -1.742 & 0.082 & -0.678 & 0.378 & -1.795 & 0.073 & 10.65 & 0.434 & 24.51 & $0.000^{*}$ & -2.299 & 0.299 & -7.678 & $0.000^{*}$ \\
\hline FL & -0.050 & 0.241 & -0.206 & 0.837 & 0.700 & 0.291 & 2.400 & $0.017^{*}$ & -0.636 & 0.188 & -3.383 & $0.001^{*}$ & -0.806 & 0.239 & -3.367 & $0.001 *$ \\
\hline FA & -0.049 & 0.068 & -0.731 & 0.465 & 0.001 & 0.001 & 1.203 & 0.230 & -0.400 & 0.052 & -7.705 & $0.000 *$ & -0.001 & 0.001 & -2.209 & $0.028 *$ \\
\hline $\mathrm{R}^{2}$ & & & & 100440 & & & & 0.045645 & & & & .633726 & & & & 0.152917 \\
\hline $\begin{array}{l}\text { Adjusted } \\
\mathrm{R}^{2}\end{array}$ & & & & 087981 & & & & 0.035927 & & & & 630198 & & & & 0.144970 \\
\hline $\begin{array}{l}\text { S.E. of } \\
\text { regression }\end{array}$ & & & & 104291 & & & & 0.842035 & & & & .045950 & & & & 0.743483 \\
\hline
\end{tabular}

From Table 5, it is demonstrated that there is no significantly association of eVAIC with firm growth rate ( $\beta=0.061, P=0.514)$ but eVAIC is significantly $(P=0.007)$ and negatively $(\beta=-0.168)$ related to the dividend payout ratio. It shows positive relationship with $\mathrm{EP}(\beta=0.813, P=0.000)$ and return on capital employed $(\beta=0.248$, $P=0.000)$. So, null hypothesis $\mathrm{H}_{1}$ is rejected. Findings proved the significance of IC in the achievement of firms. In this age, intangible assets are also required to gain competitive advantage and to attain highest profits. Nevertheless increase in investment on IC can minimize the dividend payout ratio. FRQ has a strong positive effect on firm growth rate $(\beta=0.263, P=0.000)$ and employee productivity $(\beta=0.098, P=0.002)$. However, it has an insignificant effect on dividend payout ratio $(\beta=-0.048, P=0.071)$ and return on capital employed $(\beta=0.007$, $P=0.744)$.

Table 6: Regression Analysis for Model 5, Model 6, Model 7 and Model 8

\begin{tabular}{|c|c|c|c|c|c|c|c|c|c|c|c|c|c|c|c|c|}
\hline & & & $\mathrm{GR}$ & & & & $\mathrm{PR}$ & & & & $P$ & & & & $\overline{C E}$ & \\
\hline Variables & $\beta$ & $\begin{array}{l}\text { Std. } \\
\text { Error }\end{array}$ & $\begin{array}{l}\text { t- } \\
\text { Statistic }\end{array}$ & $\mathrm{p}$ & $\beta$ & $\begin{array}{l}\text { Std. } \\
\text { Error }\end{array}$ & $\begin{array}{l}\text { t- } \\
\text { Statistic }\end{array}$ & $\mathrm{p}$ & $\beta$ & $\begin{array}{l}\text { Std. } \\
\text { Error }\end{array}$ & $\begin{array}{l}\mathrm{t}- \\
\text { Statistic }\end{array}$ & $\mathrm{p}$ & $\beta$ & $\begin{array}{l}\text { Std. } \\
\text { Error }\end{array}$ & $\begin{array}{l}\text { t- } \\
\text { Statistic }\end{array}$ & $\mathrm{p}$ \\
\hline Constant & -0.047 & 1.723 & -0.027 & 0.978 & 6.227 & 1.244 & 5.005 & $0.000^{8}$ & -22.64 & 1.465 & -15.45 & $0.000^{8}$ & 5.938 & 0.948 & 6.260 & $0.000^{8}$ \\
\hline $\mathrm{HC}$ & -0.001 & 0.068 & -0.017 & 0.986 & 1.220 & 0.717 & 1.702 & 0.089 & -0.204 & 0.057 & -3.598 & $0.000^{*}$ & 1.169 & 0.606 & 1.929 & 0.054 \\
\hline $\mathrm{RC}$ & -0.112 & 0.104 & -1.081 & 0.281 & -1.822 & 0.617 & -2.953 & $0.003^{8}$ & -0.145 & 0.087 & -1.656 & 0.098 & 0.127 & 0.515 & 0.246 & 0.806 \\
\hline $\operatorname{InC}$ & -0.136 & 0.085 & -1.600 & 0.110 & 0.183 & 0.051 & 3.590 & $0.000^{8}$ & 0.217 & 0.073 & 2.959 & $0.003^{8}$ & 0.126 & 0.043 & 2.905 & $0.004^{*}$ \\
\hline $\operatorname{PrC}$ & 0.094 & 0.099 & 0.947 & 0.344 & -0.144 & 0.062 & -2.318 & $0.021^{8}$ & -0.321 & 0.082 & -3.929 & $0.000^{8}$ & 0.147 & 0.052 & 2.810 & $0.005^{*}$ \\
\hline FRQ & 0.252 & 0.043 & 5.872 & $0.000^{*}$ & -0.031 & 0.027 & -1.145 & 0.253 & 0.115 & 0.034 & 3.330 & $0.001^{*}$ & 0.012 & 0.022 & 0.554 & 0.580 \\
\hline FS & -1.021 & 0.560 & -1.823 & 0.069 & -0.828 & 0.388 & -2.135 & $0.033^{8}$ & 10.17 & 0.477 & 21.30 & $0.000^{8}$ & -2.512 & 0.300 & -8.383 & $0.000^{8}$ \\
\hline FL & -0.087 & 0.251 & -0.347 & 0.729 & 0.966 & 0.299 & 3.231 & $0.001^{*}$ & -0.411 & 0.213 & -1.928 & $0.054^{*}$ & -0.891 & 0.243 & -3.671 & $0.000^{8}$ \\
\hline FA & -0.063 & 0.069 & -0.910 & 0.363 & 0.001 & 0.001 & 1.093 & 0.275 & -0.388 & 0.057 & -6.813 & $0.000^{*}$ & -0.002 & 0.001 & -2.837 & $0.005^{*}$ \\
\hline $\mathrm{R}^{2}$ & & & & 0.121718 & & & & 0.073983 & & & & 0.585472 & & & & 0.192509 \\
\hline $\begin{array}{l}\text { Adjusted } \\
\mathrm{R}^{2}\end{array}$ & & & & 0.102254 & & & & 0.058803 & & & & 0.579046 & & & & 0.180321 \\
\hline $\begin{array}{l}\text { S.E. of } \\
\text { regression }\end{array}$ & & & & 1.101511 & & & & 0.831984 & & & & 1.115948 & & & & 0.727952 \\
\hline
\end{tabular}


From Table 6, it is manifested that human capital is an insignificant predictor for firm growth rate $(\beta=-0.001$, $P=0.986)$, dividend payout ratio $(\beta=1.220, P=0.089)$ and return on capital employed $(\beta=1.169, P=0.054)$ but it has negative $(\beta=-0.204)$ and significant $(P=0.000)$ effect on employee productivity. So, null hypothesis $\mathrm{H}_{1 \mathrm{a}}$ is rejected. Qualified and Skilled labor is reflected as an asset for corporations. But if firms expensed heavily on employees or increase the number of employees and don't use human resource effectively then it can reduce our net profits and ultimately minimize employee productivity. Because it's not enough to have skilled personnel only, they should also be committed and motivated to contributing to their firms (Andreeva \& Garanina, 2016). Relational capital has no association with FGR $(\beta=-0.112, P=0.281)$, employee productivity $(\beta=-0.145, P=0.098)$ and returns on capital employed $(\beta=0.127, P=0.806)$ but results showed relational capital has negative association with dividend payout ratio $(\beta=-1.822, P=0.003)$. So, we null hypothesis $\mathrm{H}_{1 \mathrm{~b}}$ is rejected. For receiving high relational capital, an organization raises marketing, distribution and selling expenditures. By growing expenses, profits will be reduced so EPS and DPR will also be minimized. Moreover, there is no relationship between innovation capital and firm growth rate $(\beta=-0.136, P=0.110)$ nevertheless, innovation capital is positively linked with the dividend payout ratio $(\beta=0.183, P=0.000)$, employee productivity $(\beta=0.217, P=0.003)$ and return on capital employed ( $\beta=0.126, P=0.004)$. So, null hypothesis $\mathrm{H}_{1 \mathrm{c}}$ is rejected. $\mathrm{R} \& \mathrm{D}$ activities are necessary to bring innovation in services and products of corporations. Innovation increases operating profit and the net profit of manufacturing firms. Process capital seems to have no significant influence on firm growth rate $(\beta=0.094, P=0.344)$ and it has a strong negative effect on dividend payout ratio $(\beta=-0.144, P=0.021)$ and employee productivity $(\beta=-0.321, P=0.000)$. However, it has positive $(\beta=0.147)$ and significant $(P=0.005)$ effect on ROCE. So, null hypothesis $\mathrm{H}_{1 \mathrm{~d}}$ is rejected. If the firms have best processes, programs and techniques then organization generates high operating profit. if we expensed more in process capital then profit will reduce and eventually DPR will also minimize. In addition to, FRQ has significantly positive impact on $\operatorname{FGR}(\beta=0.252, P=0.000)$ and employee productivity $(\beta=0.115, P=0.001)$. On the contrary, it has no association with $\operatorname{DPR}(\beta=-0.031, P=0.253)$ and the return on capital employed $(\beta=0.012$, $P=0.580$ ). So, null hypothesis $\mathrm{H}_{2}$ is rejected. Accuracy, fairness and transparency of figures and facts in financial statements are also the reason for higher financial performance. Firm age, firm leverage and firm size have no significant effect on FGR. Firm size negatively and firm leverage positively influences dividend payout ratio. Firm age has no significant effect on the DPR. Firm size positively and firm leverage and firm age negatively influence employee productivity. Firm age, firm leverage and firm size negatively influence return on capital employed.

Table 7: Moderation Analysis

\begin{tabular}{ccccc}
\hline & Coefficient & Std. Error & t-Statistic & $\mathrm{p}$ \\
\hline Firm Growth Rate & & & & 0.662 \\
eVAIC $\times$ GFC & -0.001 & 0.002 & -0.437 & 0.903 \\
FRQ $\times$ GFC & 0.034 & 0.283 & 0.122 & 0.857 \\
Dividend Payout Ratio & & & & 0.997 \\
eVAIC $\times$ GFC & 2.561 & 14.17 & 0.181 & 0.727 \\
FRQ $\times$ GFC & 9.122 & 2291.3 & & 0.662 \\
Employee productivity & & & 0.349 & $0.000 *$ \\
eVAIC $\times$ GFC & 53.49 & 153.3 & 0.437 & 0.133 \\
FRQ $\times$ GFC & 10788.6 & 24698.8 & & \\
Return on Capital Employed & & & 0.916 & -1.503 \\
eVAIC $\times$ GFC & 0.887 & 0.128 & 21.66 & \\
FRQ $\times$ GFC & -32.54 & & & \\
\hline
\end{tabular}

Note: GFC $=$ Global Financial Crisis 2007-09, Interaction Term eVAIC $=$ eVAIC $\times$ GFC, Interaction Term $\mathrm{FRQ}=\mathrm{FRQ} \times \mathrm{GFC},{ }^{*} \mathrm{p}<0.05$

Table 7 indicated that Global Financial Crisis positively moderates the relationship of eVAIC on return on capital employed $(\beta=0.887, P=0.000)$. However, it does not moderate the relationship of eVAIC on firm growth rate $(\beta=-0.001, P=0.662)$, dividend payout ratio $(\beta=2.561, P=0.857)$ and employee productivity $(\beta=53.49$, $P=0.727)$. So, null hypothesis $\mathrm{H}_{3}$ is rejected. Global Financial Crisis does not moderate the relationship of FRQ on firm growth rate $(\beta=0.034, P=0.903)$, dividend payout ratio $(\beta=9.122, P=0.997)$, employee productivity $(\beta=10788.6, P=0.662)$ and return on capital employed $(\beta=-32.54, P=0.133)$. So, null hypothesis $\mathrm{H}_{4}$ is accepted.

\section{Conclusion and future direction}

This is the empirical study to examine the effect of financial reporting quality, intellectual capital and components of intellectual capital on the financial performance of fortune 500 companies. Elements of intellectual capital are relational capital, human capital, process capital and innovation capital. Results proved that intellectual capital has a significant effect on financial performance. Findings are consistent with previous studies (Khalique et al., 2015; Ferreira \& Franco, 2017). Human capital has a significant and negative effect on financial performance. Relational capital has a significant negative effect on financial performance. Finding is consistent with a study of Russia (Andreeva \& Garanina, 2016). Process capital and innovation capital has a significant effect on financial 
performance. These results enhance prior studies (Naranjo-Valencia et al., 2016; Phusavat et al., 2013; Lee \& Lin, 2018). Financial Reporting Quality has a significant positive effect on financial performance. Result enhances prior research (Martinez-Ferrero, 2014; Salehi et al., 2018). Global Financial Crisis 2007-09 positively moderates the effect of intellectual capital on financial performance but it does not the moderate the effect of financial reporting quality on financial performance. Scholar can also consider financial distress as moderating variable. All remaining industries from fortune 500 companies list should be tested for this research model.

\section{References}

Abdullah, D. F., \& Sofian, S. (2012). The relationship between intellectual capital and corporate performance. Procedia-Social and Behavioral Sciences, 40, 537-541. https://doi.org/10.1016/j.sbspro.2012.03.227

Alipour, M. (2012). The effect of intellectual capital on firm performance: an investigation of Iran insurance companies. Measuring Business Excellence, 16(1), 53-66. https://doi.org/10.1108/13683041211204671

Al-Musali, M. A., \& Ku Ismail, K. N. I. (2016). Cross-country comparison of intellectual capital performance and its impact on financial performance of commercial banks in GCC countries. International Journal of Islamic and Middle Eastern Finance and Management, 9(4), 512-531. https://doi.org/10.1108/IMEFM-03-20150029

Andreeva, T., \& Garanina, T. (2016). Do all elements of intellectual capital matter for organizational performance? Evidence from Russian context. Journal of Intellectual Capital, 17(2), 397-412. https://doi.org/10.1108/JIC-07-2015-0062

Asiaei, K., \& Jusoh, R. (2015). A multidimensional view of intellectual capital: the impact on organizational performance. Management Decision, 53(3), 668-697. https://doi.org/10.1108/MD-05-2014-0300

Atalay, M., Anafarta, N., \& Sarvan, F. (2013). The relationship between innovation and firm performance: An empirical evidence from Turkish automotive supplier industry. Procedia-Social and Behavioral Sciences, 75, 226-235. https://doi.org/10.1016/j.sbspro.2013.04.026

Bartram, S. M., \& Bodnar, G. M. (2009). No place to hide: The global crisis in equity markets in 2008/2009. Journal of international Money and Finance, 28(8), 1246-1292. https://doi.org/10.1016/j.jimonfin.2009.08.005

Bhatt, R. R., \& Bhattacharya, S. (2015). Board structure and firm performance in Indian IT firms. Journal of Advances in Management Research, 12(3), 232-248. https://doi.org/10.1108/JAMR-07-2014-0042

Biddle, G. C., Hilary, G., \& Verdi, R. S. (2009). How does financial reporting quality relate to investment efficiency?. Journal of accounting and economics, 48(2-3), 112-131. https://doi.org/10.1016/j.jacceco.2009.09.001

Bontis, N. (2001). Assessing knowledge assets: a review of the models used to measure intellectual capital. International journal of management reviews, 3(1), 41-60. https://doi.org/10.1111/1468-2370.00053

Breusch, T. S., \& Pagan, A. R. (1979). A simple test for heteroscedasticity and random coefficient variation. Econometrica: Journal of the Econometric Society, 1287-1294. https://www.jstor.org/stable/1911963

Brooking, A. (1996). Intellectual Capital: Core Assets for the Third Millennium Enterprise, International Thompson Business Press, London.

Cabrita, M. D. R., \& Bontis, N. (2008). Intellectual capital and business performance in the Portuguese banking industry. International Journal of Technology Management, 43(1-3), 212-237. https://doi.org/10.1504/IJTM.2008.019416

Cecchetti, S. G. (2009). Crisis and responses: the Federal Reserve in the early stages of the financial crisis. Journal of Economic Perspectives, 23(1), 51-75. https://doi.org/10.1257/jep.23.1.51

Celenza, D., \& Rossi, F. (2014). Intellectual capital and performance of listed companies: empirical evidence from Italy. Measuring Business Excellence, 18(1), 22-35. https://doi.org/10.1108/MBE-10-2013-0054

Charbaji, A. (2011). Applied Econometrics Using Eviews, SPSS And Excel With Applications In Arab Countries: Author House Publishing.

Chen, F., Hope, O. K., Li, Q., \& Wang, X. (2011). Financial reporting quality and investment efficiency of private firms in emerging markets. The accounting review, 86(4), 1255-1288. https://doi.org/10.2308/accr-10040

Chen, F. C., Liu, Z. J., \& Kweh, Q. L. (2014). Intellectual capital and productivity of Malaysian general insurers. Economic Modelling, 36, 413-420. https://doi.org/10.1016/j.econmod.2013.10.008

Chen, J., Zhu, Z., \& Yuan Xie, H. (2004). Measuring intellectual capital: a new model and empirical study. Journal of Intellectual capital, 5(1), 195-212. https://doi.org/10.1108/14691930410513003

Cheng, M. Y., Lin, J. Y., Hsiao, T. Y., \& Lin, T. W. (2010). Invested resource, competitive intellectual capital, and corporate performance. Journal of intellectual capital, 11(4), 433-450. https://doi.org/10.1108/14691931011085623

Chiou, C. C., \& Chen, Y. C. (2012). Relations among learning orientation, innovation capital and firm performance: An empirical study in Taiwan's IT/electronic industry. International Journal of Management, 29(3), 321.

Clarke, M., Seng, D., \& Whiting, R. H. (2011). Intellectual capital and firm performance in Australia. Journal of 
Intellectual Capital, 12(4), 505-530. https://doi.org/10.1108/14691931111181706

Cohen, S., \& Kaimenakis, N. (2007). Intellectual capital and corporate performance in knowledge-intensive SMEs. The Learning Organization, 14(3), 241-262. https://doi.org/10.1108/09696470710739417

Colombelli, A. (2015). Top management team characteristics and firm growth: Evidence from a sample of listed companies. International Journal of Entrepreneurial Behavior \& Research, 21(1), 107-127. https://doi.org/10.1108/IJEBR-10-2013-0181

Cousins, P. D., Handfield, R. B., Lawson, B., \& Petersen, K. J. (2006). Creating supply chain relational capital: the impact of formal and informal socialization processes. Journal of operations management, 24(6), 851863. https://doi.org/10.1016/j.jom.2005.08.007

Dechow, P. M., \& Dichev, I. D. (2002). The quality of accruals and earnings: The role of accrual estimation errors. The accounting review, 77(s-1), 35-59. https://doi.org/10.2308/accr.2002.77.s-1.35

Díaz-Fernández, M. C., González-Rodríguez, M. R., \& Simonetti, B. (2015). Top management team’s intellectual capital and firm performance. European Management Journal, 33(5), 322-331. https://doi.org/10.1016/j.emj.2015.03.004

Ding, Y. (2010). Study on the management of intellectual capital. International Journal of Business and Management, 5(2), 213-216. https://10.5539/ijbm.v5n2p213

Dwyer, G. P., \& Lothian, J. R., (2012). International and historical dimensions of the financial crisis of 2007 and 2008. Journal of International Money and Finance, 31(1), 1-9. https://doi.org/10.1016/j.jimonfin.2011.11.006

Edvinsson, L., \& Malone, M. S. (1997). Intellectual capital: realizing your company/'s true value by finding its hidden brainpower. http://www.citeulike.org/group/8343/article/1868716

El-Bannany, M. (2012). Global financial crisis and the intellectual capital performance of UAE banks. Journal of Human Resource Costing \& Accounting, 16(1), 20-36. https://doi.org/10.1108/14013381211272626

Enekwe, C. I., Nweze, A. U., \& Agu, C. (2015). The effect of dividend payout on performance evaluation: Evidence of quoted cement companies in Nigeria. European Journal of Accounting, Auditing and Finance Research, 3(11), 40-59.

Erkens, D. H., Hung, M., \& Matos, P. (2012). Corporate governance in the 2007-2008 financial crisis: Evidence from financial institutions worldwide. Journal of Corporate Finance, 18(2), 389-411. https://doi.org/10.1016/j.jcorpfin.2012.01.005

Ferraro, O., \& Veltri, S. (2011). The value relevance of intellectual capital on the firm's market value: an empirical survey on the Italian listed firms. International Journal of Knowledge-Based Development, 2(1), 66-84. https://ssrn.com/abstract=2187229

Ferreira, A., \& Franco, M. (2017). The Mediating Effect of Intellectual Capital in The Relationship Between Strategic Alliances and Organizational Performance in Portuguese Technology-Based SMEs. European Management Review, 14(3), 303-318. https://doi.org/10.1111/emre.12107

Gogan, L. M., Artene, A., Sarca, I., \& Draghici, A. (2016). The impact of intellectual capital on organizational performance. Procedia-social and behavioral sciences, 221, 194-202. https://doi.org/10.1016/j.sbspro.2016.05.106

Hair, J. F., Black, W. C., Babin, B. J., Anderson, R. E., \& Tatham, R. L. (2006). Multivariate data analysis.

Hamdan, A. (2018). Intellectual capital and firm performance: Differentiating between accounting-based and market-based performance. International Journal of Islamic and Middle Eastern Finance and Management, 11(1), 139-151. https://doi.org/10.1108/IMEFM-02-2017-0053

Hashim, M. J., Osman, I., \& Alhabshi, S. M. (2015). Effect of intellectual capital on organizational performance. Procedia-Social and Behavioral Sciences, 211, 207-214. https://doi.org/10.1016/j.sbspro.2015.11.085

Honu, M. V., \& Gajevszky, A. (2014). THE QUALITY OF FINANCIAL REPORTING AND CORPORATE GOVERNANCE: EVIDENCE FROM ROMANIAN'S AERONAUTIC INDUSTRY. Economic and Social Development: Book of Proceedings, 517.

Iatridis, G. (2010). International Financial Reporting Standards and the quality of financial statement information. International Review of Financial Analysis, 19(3), 193-204. https://doi.org/10.1016/j.irfa.2010.02.004

Inkinen, H. (2015). Review of empirical research on intellectual capital and firm performance. Journal of Intellectual capital, 16(3), 518-565. https://doi.org/10.1108/JIC-01-2015-0002

Jardon, C. M., \& Dasilva, A. (2017). Intellectual capital and environmental concern in subsistence small businesses. Management of Environmental Quality: An International Journal, 28(2), 214-230. https://doi.org/10.1108/MEQ-05-2015-0085

Jiang, L. A., Waller, D. S., \& Cai, S. (2013). Does ownership type matter for innovation? Evidence from China. Journal of Business Research, 66(12), 2473-2478. https://doi.org/10.1016/j.jbusres.2013.05.037

Jordão, R. V. D., \& Almeida, V. R. D. (2017). Performance measurement, intellectual capital and financial sustainability. Journal of Intellectual Capital, 18(3), 643-666. https://doi.org/10.1108/JIC-11-2016-0115

Kachouri, M., \& Jarboui, A. (2017). Exploring the relation between corporate reporting and corporate governance 
effectiveness. Journal of Financial Reporting and Accounting, 15(3), 347-366. https://doi.org/10.1108/JFRA-06-2016-0053

Kai Wah Chu, S., Hang Chan, K., \& Wu, W. W. (2011). Charting intellectual capital performance of the gateway to China. Journal of Intellectual Capital, 12(2), 249-276. https://doi.org/10.1108/14691931111123412

Kardan, B., Salehi, M., \& Abdollahi, R. (2016). The relationship between the outside financing and the quality of financial reporting: evidence from Iran. Journal of Asia Business Studies, 10(1), 20-40. https://doi.org/10.1108/JABS-04-2014-0027

Kehelwalatenna, S. (2016). Intellectual capital performance during financial crises. Measuring Business Excellence, 20(3), 55-78. https://doi.org/10.1108/MBE-08-2015-0043

Kennedy, P. (1985). A Guide to Econometrics, 2nd ed., The MIT Press, Cambridge, MA.

Khalique, M., Bontis, N., Abdul Nassir bin Shaari, J., \& Hassan Md. Isa, A. (2015). Intellectual capital in small and medium enterprises in Pakistan. Journal of Intellectual Capital, 16(1), 224-238. https://doi.org/10.1108/JIC-01-2014-0014

Koo, D. S., Ramalingegowda, S., \& Yu, Y. (2017). The effect of financial reporting quality on corporate dividend policy. Review of Accounting Studies, 22(2), 753-790. https://doi.org/10.1007/s11142-017-9393-3

Krismiaji, Y., Aryani, A., \& Suhardjanto, D. (2016). International Financial Reporting Standards, board governance, and accounting quality: A preliminary Indonesian evidence. Asian Review of Accounting, 24(4), 474-497. https://doi.org/10.1108/ARA-06-2014-0064

Laitinen, E. K. (2009). Importance of performance information in managerial work. Industrial Management \& Data Systems, 109(4): 550-569. https://doi.org/10.1108/02635570910948669

Lee, C. C., \& Lin, C. K. (2018). The major determinants of influencing the operating performance from the perspective of intellectual capital: Evidence on CPA industry. Asia Pacific Management Review. https://doi.org/10.1016/j.apmrv.2018.01.006

Lee, I. C., Lin, C. Y., \& Lin, T. Y. (2017). The creation of national intellectual capital from the perspective of Hofstede's national culture. Journal of Intellectual Capital, 18(4), 807-831. https://doi.org/10.1108/JIC-112016-0117

Lin, C. J., Wang, T., \& Pan, C. J. (2016). Financial reporting quality and investment decisions for family firms. Asia Pacific Journal of Management, 33(2), 499-532. https://doi.org/10.1007/s10490-015-9438-8

Lin, Z., Jiang, Y., Tang, Q., \& He, X. (2014). Does high-quality financial reporting mitigate the negative impact of global financial crises on firm performance? Evidence from the United Kingdom. Australasian Accounting, Business and Finance Journal, 8(5), 19-46. http://hdl.handle.net/10453/34099

Lins, K. V., Servaes, H., \& Tamayo, A. (2017). Social capital, trust, and firm performance: The value of corporate social responsibility during the financial crisis. The Journal of Finance, 72(4), 1785-1824. https://doi.org/10.1111/jofi.12505

Lobo, G. J., Xie, Y., \& Zhang, J. H. (2018). Innovation, financial reporting quality, and audit quality. Review of Quantitative Finance and Accounting, 51(3), 719-749. https://doi.org/10.1007/s11156-017-0686-1

Maddala, G. S., \& Wu, S. (1999). A comparative study of unit root tests with panel data and a new simple test. Oxford Bulletin of Economics and statistics, 61(S1), 631-652. https://doi.org/10.1111/1468-0084.0610s1631

Maditinos, D., Chatzoudes, D., Tsairidis, C., \& Theriou, G. (2011). The impact of intellectual capital on firms' market value and financial performance. Journal of intellectual capital, 12(1), 132-151. https://doi.org/10.1108/14691931111097944

Maditinos, D., Sevic, Z., \& Tsairidis, C. (2010). Intellectual capital and business performance: an empirical study for the Greek listed companies. European Research Studies, 13(3), 145-168. https://ersj.eu/journal/291\#

Maji, S. G., \& Goswami, M. (2016). Intellectual capital and firm performance in emerging economies: the case of India. Review of International Business and Strategy, 26(3), 410-430. https://doi.org/10.1108/RIBS-032015-0019

Maria Morariu, C. (2014). Intellectual capital performance in the case of Romanian public companies. Journal of Intellectual Capital, 15(3), 392-410. https://doi.org/10.1108/JIC-05-2014-0061

Martínez-Ferrero, J. (2014). Consequences of financial reporting quality on corporate performance: Evidence at the international level. Estudios de Economía, 41(1), 49-88. http://hdl.handle.net/10419/146717

Mention, A. L., \& Bontis, N. (2013). Intellectual capital and performance within the banking sector of Luxembourg and Belgium. Journal of Intellectual capital, 14(2), 286-309. https://doi.org/10.1108/14691931311323896

Mohammadi, S. M. (2014). The relationship between financial reporting quality and investment efficiency in Tehran stock exchange. International Journal of Academic Research in Business and Social Sciences, 4(6), 104. http://dx.doi.org/10.6007/IJARBSS/v4-i6/930

Mondal, A., \& Ghosh, S. K. (2012). Intellectual capital and financial performance of Indian banks. Journal of Intellectual Capital, 13(4), 515-530. https://doi.org/10.1108/14691931211276115

Namvar, M., Fathian, M., Gholamin, M., \& Akhavan, P. (2012). Exploring the role of human capital on firm's structural capital in Iranian e-business industry. http://dx.doi.org/10.2139/ssrn.2187267 
Naranjo-Valencia, J. C., Jiménez-Jiménez, D., \& Sanz-Valle, R. (2016). Studying the links between organizational culture, innovation, and performance in Spanish companies. Revista Latinoamericana de Psicología, 48(1), 30-41. https://doi.org/10.1016/j.rlp.2015.09.009

Nazari, J. A., \& Herremans, I. M. (2007). Extended VAIC model: measuring intellectual capital components. Journal of Intellectual Capital, 8(4), 595-609. https://doi.org/10.1108/14691930710830774

Nimtrakoon, S. (2015). The relationship between intellectual capital, firms' market value and financial performance: Empirical evidence from the ASEAN. Journal of Intellectual Capital, 16(3), 587-618. https://doi.org/10.1108/JIC-09-2014-0104

Ozkan, N., Cakan, S., \& Kayacan, M. (2017). Intellectual capital and financial performance: A study of the Turkish Banking Sector. Borsa Istanbul Review, 17(3), 190-198. https://doi.org/10.1016/j.bir.2016.03.001

Peek, E., Cuijpers, R., \& Buijink, W. (2010). Creditors' and shareholders' reporting demands in public versus private firms: Evidence from Europe. Contemporary Accounting Research, 27(1), 49-91. https://doi.org/10.1111/j.1911-3846.2010.01001.x

Phusavat, K., Comepa, N., Sitko-Lutek, A., \& Ooi, K. B. (2011). Interrelationships between intellectual capital and performance: Empirical examination. Industrial Management \& Data Systems, 111(6), 810-829. https://doi.org/10.1108/02635571111144928

Phusavat, K., Comepa, N., Sitko-Lutek, A., \& Ooi, K. B. (2012). Intellectual capital: national implications for industrial competitiveness. Industrial Management \& Data Systems, 112(6), 866-890. https://doi.org/10.1108/02635571211238491

Phusavat, K., Comepa, N., Sitko-Lutek, A., \& Ooi, K. B. (2013). Productivity management: integrating the intellectual capital. Industrial Management \& Data Systems, 113(6), 840-855. https://doi.org/10.1108/IMDS-09-2012-0330

Pucci, T., Simoni, C., \& Zanni, L. (2015). Measuring the relationship between marketing assets, intellectual capital and firm performance. Journal of Management \& Governance, 19(3), 589-616. https://doi.org/10.1007/s10997-013-9278-1

Pulic, A. (1998), January. Measuring the performance of intellectual potential in knowledge economy. In $2 n d$ McMaster Word Congress on Measuring and Managing Intellectual Capital by the Austrian Team for Intellectual Potential.

Reinhart, C. M., \& Rogoff, K. S. (2009). This time is different: Eight centuries of financial folly. princeton university press.

Reinhart, C. M., \& Rogoff, K. S. (2013). Banking crises: an equal opportunity menace. Journal of Banking \& Finance, 37(11), 4557-4573. https://doi.org/10.1016/j.jbankfin.2013.03.005

Rodrigues, L. L., Tejedo-Romero, F., \& Craig, R. (2017). Corporate governance and intellectual capital reporting in a period of financial crisis: Evidence from Portugal. International Journal of Disclosure and Governance, 14(1), 1-29. https://doi.org/10.1057/jdg.2015.20

Roos, J., Roos, G., Edvinsson, L., \& Dragonetti, N. C. (1998). Intellectual Capital: Navigating the New Business Landscape, New York University Press, New York, NY.

Ruiqi, W., Wang, F., Xu, L., \& Yuan, C. (2017). R\&D expenditures, ultimate ownership and future performance: Evidence from China. Journal of Business Research, 71, 47-54. https://doi.org/10.1016/j.jbusres.2016.10.018

Salehi, M., \& Shirazi, M. (2016). Audit committee impact on the quality of financial reporting and disclosure: Evidence from the Tehran stock exchange. Management Research Review, 39(12), 1639-1662. https://doi.org/10.1108/MRR-09-2015-0198

Salehi, M., Enayati, G., \& Javadi, P. (2014). The relationship between intellectual capital with economic value added and financial performance. Iranian Journal of Management Studies, 7(2), 259-283. https://ijms.ut.ac.ir/article 36618 4692.html

Salehi, M., Tagribi, M., \& Farhangdoust, S. (2018). The effect of reporting quality on stock returns of listed companies on the Tehran Stock Exchange. International Journal of Productivity and Performance Management, 67(1), 4-19. https://doi.org/10.1108/IJPPM-09-2015-0127

Sardo, F., \& Serrasqueiro, Z. (2017). A European empirical study of the relationship between firms' intellectual capital, financial performance and market value. Journal of Intellectual Capital, 18(4), 771-788. https://doi.org/10.1108/JIC-10-2016-0105

Scafarto, V., Ricci, F., \& Scafarto, F. (2016). Intellectual capital and firm performance in the global agribusiness industry: The moderating role of human capital. Journal of Intellectual Capital, 17(3), 530-552. https://doi.org/10.1108/JIC-11-2015-0096

Setiyawati, H. (2013). The effect of Internal Accountants' Competence, Managers' Commitment to Organizations and the Implementation of the Internal Control System on the Quality of Financial Reporting. International Journal of Business and Management Invention, 2(11.1927). http://www.ijbmi.org/papers/Vol(2)11/Version1/C021101019027.pdf

Shiu, H. J. (2006). The application of the value added intellectual coefficient to measure corporate performance: 
evidence from technological firms. International Journal of Management, 23(2), 356.

Singh, S., Sidhu, J., Joshi, M., \& Kansal, M. (2016). Measuring intellectual capital performance of Indian banks: A public and private sector comparison. Managerial Finance, 42(7), 635-655. https://doi.org/10.1108/MF08-2014-0211

Stewart, T. (1997). Intellectual Capital: The New Wealth of Organizations, Doubleday/Currency, New York, NY.

Stubben, S. R. (2010). Discretionary revenues as a measure of earnings management. The accounting review, 85(2), 695-717. https://doi.org/10.2308/accr.2010.85.2.695

Su, H. Y., Fang, S. C., \& Young, C. S. (2013). Influences of relationship transparency from intellectual capital reporting on supply chain partnerships with suppliers: a field experiment. Supply Chain Management: An International Journal, 18(2), 178-193. https://doi.org/10.1108/13598541311318818

Sumedrea, S. (2013). Intellectual capital and firm performance: A dynamic relationship in crisis time. Procedia Economics and Finance, 6, 137-144. https://doi.org/10.1016/S2212-5671(13)00125-1

Vander Bauwhede, H., De Meyere, M., \& Van Cauwenberge, P. (2015). Financial reporting quality and the cost of debt of SMEs. Small Business Economics, 45(1), 149-164. https://doi.org/10.1007/s11187-015-9645-1

Vazifehdoust, H., Khajenasir, M., \& Karami, H. (2014). A study on the effect of intellectual capital on firm performance: Evidence from Tehran Stock Exchange. Management Science Letters, 4(3), 559-566.

Vithessonthi, C., \& Tongurai, J. (2015). The effect of firm size on the leverage-performance relationship during the financial crisis of 2007-2009. Journal of Multinational Financial Management, 29, 1-29. https://doi.org/10.1016/j.mulfin.2014.11.001

Wang, Z., Wang, N., \& Liang, H. (2014). Knowledge sharing, intellectual capital and firm performance. Management decision, 52(2), 230-258. https://doi.org/10.1108/MD-02-2013-0064

Yasser, Q. R., Mamun, A. A., \& Ahmed, I. (2016). Quality of financial reporting in the Asia-Pacific region: The influence of ownership composition. Review of International Business and Strategy, 26(4), 543-560. https://doi.org/10.1108/RIBS-02-2016-0011

Yasser, Q. R., Mamun, A. A., \& Hook, M. (2017). The impact of ownership structure on financial reporting quality in the east. International Journal of Organizational Analysis, 25(2), 178-197. https://doi.org/10.1108/IJOA08-2015-0894

Zakery, A., \& Afrazeh, A. (2015). Intellectual capital based performance improvement, study in insurance firms. Journal of Intellectual Capital, 16(3), 619-638. https://doi.org/10.1108/JIC-06-2014-0067 